\title{
Improved QRS Detection in Pediatric Stress Test Electrocardiograms
}

\author{
M Findeis $^{1}$, W Kaiser ${ }^{1}$, BJ Young ${ }^{2}$ \\ ${ }^{1}$ GE Healthcare Information Technologies, Freiburg, Germany \\ ${ }^{2}$ GE Healthcare Information Technologies, Milwaukee, USA
}

\begin{abstract}
Accurate QRS complex detection during exercise stress testing continues to be a critically important and challenging task, especially for pediatric ECGs during the high exercise phase.

As part of the computerized ECG analysis program used in commercial stress testing systems, the Intelligent Lead Switch algorithm was developed to select the optimal leads for QRS detection based upon signal quality. This algorithm was optimized to improve QRS detection performance for pediatric as well as adult ECGs.

The performance of QRS detection was assessed using a database with 143 pediatric and 943 adult exercise stress test ECGs. The QRS detection and arrhythmia classification results of the new version were compared with the results from the previous version. The number of QRS detection errors was reduced by 75 percent for the pediatric ECGs and by 23 percent for the adult ECGs.
\end{abstract}

\section{Introduction}

Exercise stress test ECGs are often very noisy, especially in the high exercise phase. Muscle noise, unstable baseline and cyclic artifacts produced by footfall on treadmills or by pedaling on bicycle ergometers can cause errors in QRS detection and consequently in the heart rate calculation. The difficulties in QRS detection are even greater with pediatric ECGs because of higher variability of the heart rate and the amplitude and narrowness of QRS complexes.

\section{Methods and data}

\subsection{Intelligent Lead Switch}

The signal-processing algorithm developed by GE Healthcare for processing of stress test ECGs, takes advantage of the redundancy of the multilead system.

Within the signal-processing algorithm, the Intelligent Lead Switch is the central algorithm for QRS detection and arrhythmia analysis. It has been previously described in [1] and [2]. The Intelligent Lead Switch consists of independent and equivalent units for QRS detection, event classification (single beat arrhythmia analysis) and ECG quality level calculation for each lead; and a logic unit for selecting the results of the best lead based on the quality level, correcting the QRS trigger points of the best lead and the event classification of the best lead using the results of the second best lead.

A block diagram is shown in figure 1 .

The final results from the Intelligent Lead Switch algorithm, the QRS trigger points and the arrhythmia events, are used for further processing, e.g. the heart rate calculation.

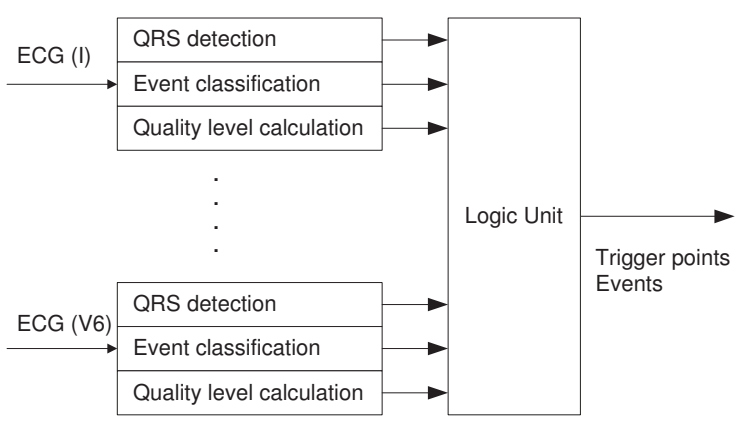

Figure 1. Block diagram of the Intelligent Lead Switch

For the processing of pediatric stress test ECGs the QRS detection, the quality level calculation and the lead selection based on the quality level were improved.

\subsubsection{QRS detection}

In pediatric exercise stress test ECGs higher heart rate variability, higher QRS amplitude variety, steeper QRS slopes and larger $\mathrm{T}$ waves than in adult stress test ECGs were found. Two examples are shown in figures 2 and 3.

The QRS trigger was improved for pediatric ECGs by enlarging the slope limit for spike detection and refining the function for the calculation of the QT-interval as a function of the RR-interval. 


\subsubsection{Quality level calculation}

For the calculation of the quality level of a lead the algorithm uses 5 parameters:

Lead status: Lead fail information.

Filtered QRS amplitude: Median of QRS amplitudes of the last 3 QRS complexes. The QRS amplitudes are calculated from the band pass filtered ECG (figure 4).

Number of cyclic artifacts: Number of 2-second intervals out of last 16 sliding intervals with cyclic artifacts.

Number of other artifacts: Number of 2-second intervals with other artifacts, e.g. muscle tremor, in a sliding window with 16 intervals.

Variance of the RR interval: $\frac{1}{8} \sum_{i=1}^{8}\left|R R_{i}-\overline{R R}\right|$, where $R R_{i}$ are the last $8 \mathrm{RR}$ intervals in the lead and $\overline{R R}$ is the average of these intervals.

Each parameter $p_{n}$ is normalized by a specific quality function $q f_{n}\left(p_{n}\right)$. The quality functions are linearly combined to calculate the quality level $q l(p)$ with $p=$ $\left(p_{1}, \ldots, p_{N}\right)$ for each lead $l d$ :

$$
q l[l d]=k_{1} * q f_{1}\left(p_{1}[l d]\right)+\ldots+k_{N} * q f_{N}\left(p_{N}[l d]\right)
$$

For practical reasons it was defined that good signal quality is indicated by a small value of $q l$. So the quality functions for the Lead status (= stat) and the Filtered QRS amplitude $(=a m p l)$ could be defined as:

$$
\begin{aligned}
& q f(\text { stat })=\left\{\begin{array}{rll}
0 & : & \text { if lead is OK } \\
\infty & : & \text { if lead is dropped off } \\
1 / & \text { ampl }
\end{array}\right. \\
& q f(\text { ampl })=
\end{aligned}
$$

For the other parameters the identity function $q f(p)=p$ is used for the normalization.

\subsubsection{Optimization of quality level function}

With the linear function $q l$ for the quality level calculation the improvement of the lead selection is reduced to the problem to find the optimal values for the factors $k_{1}, k_{2}, \ldots, k_{N}$. For this optimization a subset of the stress test database with specific examples of QRS detection errors was used. The sum of false negative and false positive detected QRS complexes was minimized by variation of $k_{1}, k_{2}, \ldots, k_{N}$. The optimal factors were implemented in the algorithm.

\subsubsection{Lead selection}

The quality level is calculated for each lead every 2 seconds. The algorithm looks for the lead with the smallest quality level. If the quality level of this lead is less than the quality level of the currently selected, the algorithm switches to this lead. A hysteresis level is used in the quality level comparison to avoid oscillations between two leads with very similar quality levels.

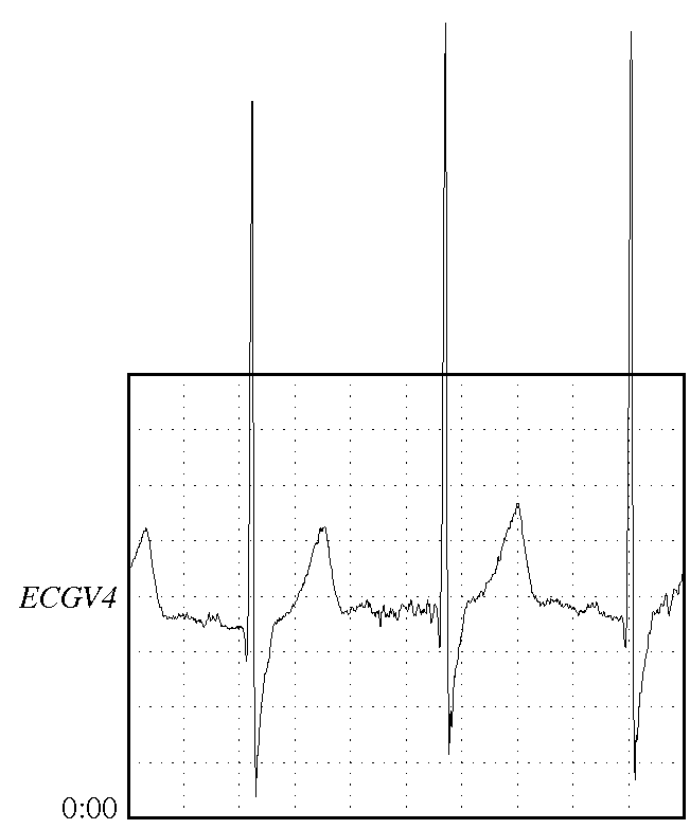

Figure 2. Pediatric ECG with QRS slopes $>1.0 \mathrm{mV} / \mathrm{ms}$, Grid: $0.2 \mathrm{sec}, 0.5 \mathrm{mV}$

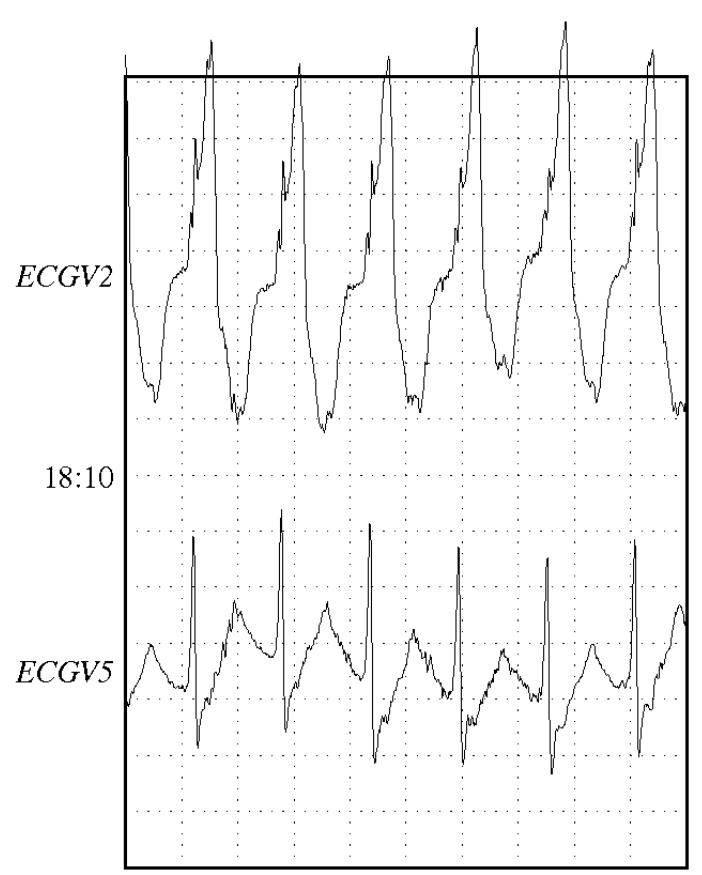

Figure 3. Pediatric ECG with large T-waves at high heart rate, Grid: $0.2 \mathrm{sec}, 0.5 \mathrm{mV}$ 


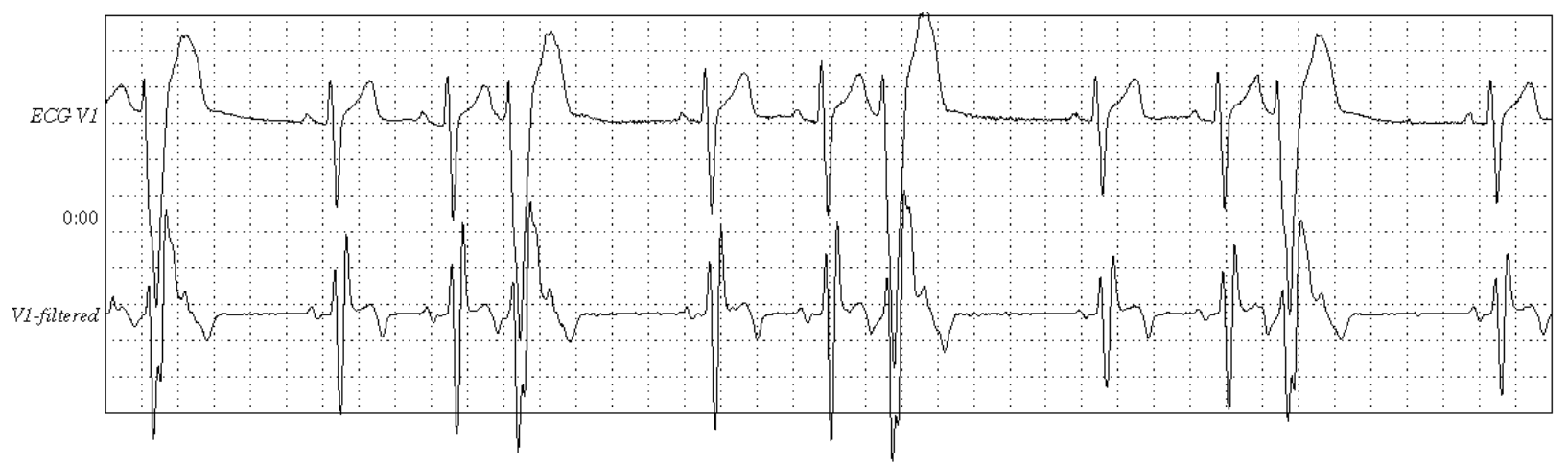

Figure 4. Original ECG (first row) and filtered ECG (second row). The difference in the amplitude between normal QRS complexes and ventricular premature beats is smaller in the filtered ECG than in the original ECG. Calculating the QRS amplitude from the filtered ECG prevents undesired lead switching due to large VPB amplitudes.

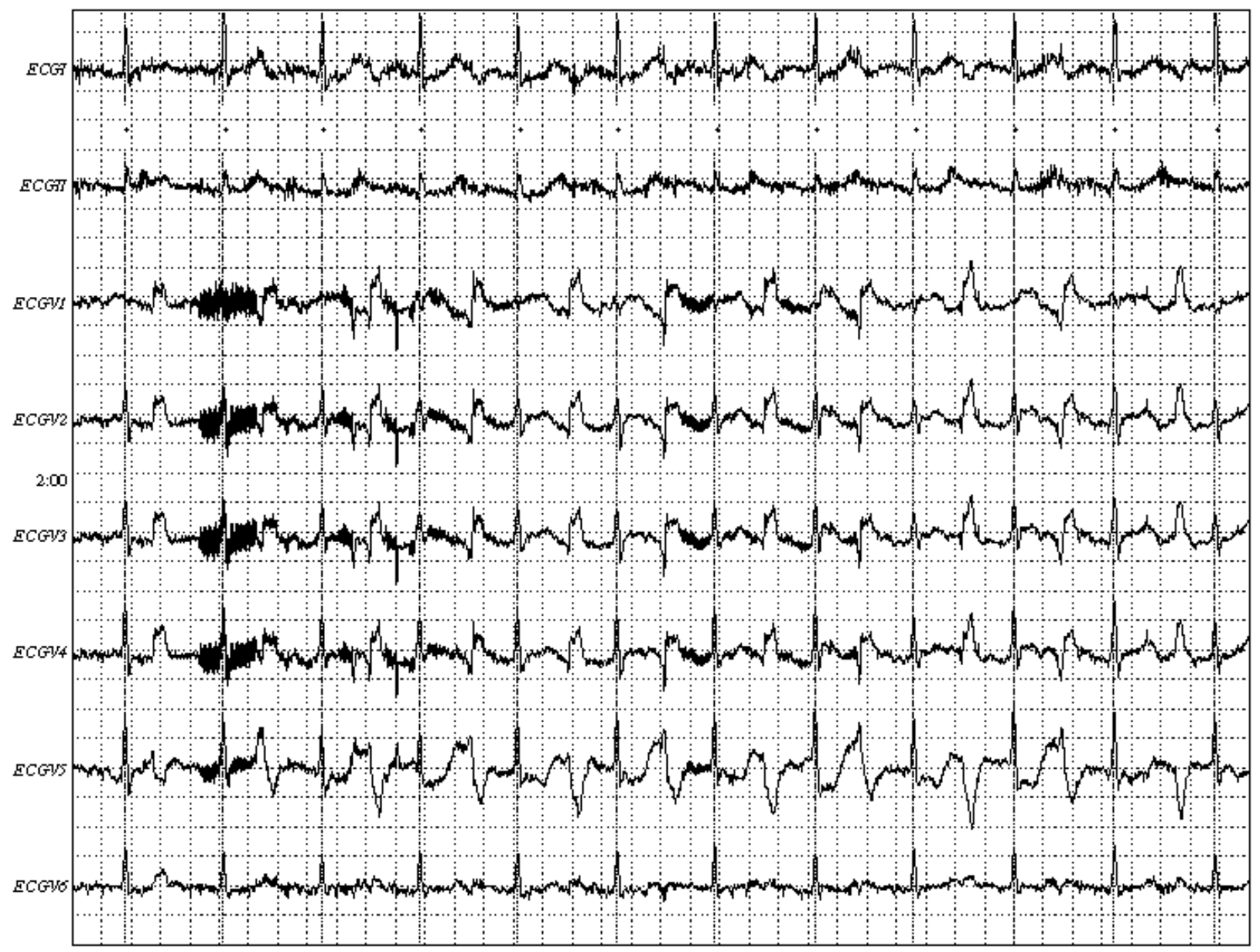

Figure 5. Stress test ECG with cyclic artifacts produced by footfall on a treadmill. Leads V1 to V5 are distorted. Only in leads I and V6 the QRS complexes can be detected properly. 


\subsection{Data}

The quality of the QRS detection was measured with an exercise stress test database containing 143 pediatric records and 943 adult records. The ECG records consist of continuous data, sampled with $500 \mathrm{~Hz}$ with a resolution of $5 \mu \mathrm{V}$. The exercise stress tests were done with treadmills (42 pediatric and 319 adult tests), and bicycle ergometers in sitting position (101 pediatric and 418 adult tests) and supine position (292 adults). The average length is $24 \mathrm{~min}$ for the pediatric ECGs and $17 \mathrm{~min}$ for the adult ECGs. The average pediatric patient age is 12 years with a range from 5 to 18 years and 56 years with a range from 18 to 87 for the adult patients. The reference QRS annotations were produced by using a first pass computer analysis, which was then corrected and verified by a human over reader.

\section{Results}

For the pediatric stress test data set with 143 records the number of QRS detection errors (sum of false positive and false negative detected QRS complexes) was reduced by $75 \%$. The QRS detection sensitivity increased from $99.84 \%$ to $99.97 \%$, and the positive predictivity also increased from $99.94 \%$ to $99.97 \%$. For the adult stress test data set with 943 records the number of QRS detection errors was reduced by $23 \%$, the QRS detection sensitivity increased from $99.91 \%$ to $99.93 \%$ while the positive predictivity increased from $99.92 \%$ to $99.94 \%$.

The results are summarized in table 1 using the following notation:

TP Number of true positive detected QRS complexes

FN Number of false negative detected QRS complexes

FP Number of false positive detected QRS complexes

QErr Number of QRS detection errors $=F N+F P$

QSe QRS detection sensitivity $=\frac{T P}{T P+F N}$

QPP QRS detection positive predictivity $=\frac{T P}{T P+F P}$

\begin{tabular}{lrrrr}
\hline & \multicolumn{2}{c}{ Pediatric ECGs } & \multicolumn{2}{c}{ Adult ECGs } \\
\hline & Old & New & Old & New \\
\hline TP & 384598 & 385110 & 1513546 & 1513915 \\
FN & 612 & 100 & 1416 & 1047 \\
FP & 213 & 107 & 1223 & 982 \\
QErr & 825 & 207 & 2639 & 2029 \\
QSe & $99.84 \%$ & $99.97 \%$ & $99.91 \%$ & $99.93 \%$ \\
QPP & $99.94 \%$ & $99.97 \%$ & $99.92 \%$ & $99.94 \%$ \\
\hline
\end{tabular}

Table 1. QRS detection results with old and new version of the algorithm.

\section{Discussion and conclusions}

The modeling of the quality level function in a linear equation facilitated the effective optimization of the coefficients for the quality level parameters. In this way a significant improvement of the QRS complex detection was achieved. Both sensitivity and positive predictivity increased, for pediatric exercise stress tests as well as for adult exercise stress tests. The improved algorithm is implemented in the GE stress test products CASE and CardioSoft.

\section{References}

[1] Kaiser W, Findeis M. Artifact processing during exercise testing. Journal of Electrocardiology 1999; 32(Supplement):212-219.

[2] Kaiser W, Findeis M. Novel signal processing methods for exercise ecg. International Journal of Bioelectromagnetism 2000;2:60-65.

Address for correspondence:

Martin Findeis

GE Healthcare Information Technologies

Munzinger Strasse 3

D-79111 Freiburg

Germany

martin.findeis@med.ge.com 